\title{
UPGRADE OF THE SLAC SLED-II PULSE COMPRESSION SYSTEM BASED ON RECENT HIGH POWER TESTS*
}

\author{
A.E.Vlieks, W.R. Fowkes, R.J.Loewen and S.G.Tantawi \\ Stanford Linear Accelerator Center, Stanford University, Stanford, CA 94309 U.S.A.
}

\begin{abstract}
In the Next Linear Collider (NLC) it is expected that the high power rf components be able to handle peak power levels in excess of $400 \mathrm{MW}$. We present recent results of high power tests designed to investigate the RF breakdown limits of the X-band pulse compression system used at SLAC. (SLED-II). Results of these tests show that both the $\mathrm{TE}_{01}-\mathrm{TE}_{10}$ mode converter and the 4-port hybrid have a maximum useful power limit of 220-250 MW. Based on these tests, modifications of these components have been undertaken to improve their peak field handling capability. Results of these modifications will be presented.
\end{abstract}

\section{INTRODUCTION}

As part of an international effort to develop a new 0.5-1.5 $\mathrm{TeV}$ electron-positron linear collider for the 21 st century, SLAC has been working towards a design, referred to as "The Next Linear Collider" (NLC), which will operate at 11.424 GHz and utilize 50 -75 MW klystrons as rf power sources. One of the major challenges in this design, or any other design, is how to generate and efficiently transport extremely high rf power from a source to an accelerator structure. SLAC has been investigating various methods of "pulse compressing" a relatively wide rf pulse $(\geq 1 \mu \mathrm{s})$ from a klystron into a narrower, but more intense, pulse. Currently a SLED-II pulse compression scheme [1] is being used at SLAC in the NLC Test Accelerator (NLCTA) and in the Accelerator Structures Test Area (ASTA) to provide high rf power for accelerator and component testing. In ASTA, a $1.05 \mu \mathrm{s}$ pulse from a 50 MW klystron was successfully pulse compressed to $205 \mathrm{MW}$ with a pulse width of $150 \mathrm{~ns}$.

Since operation in NLC will require generating and transporting rf power in excess of $400 \mathrm{MW}$ it was decided to test the breakdown limits of the SLED-II rf components in ASTA with rf power up to the maximum available of $400 \mathrm{MW}$. This required the combining of power from two $50 \mathrm{MW}$ klystrons and feeding the summed power into the SLED-II pulse compressor. Results from this experiment demonstrated that two of the key components of SLED-II; the Magic Tee and the "Flower Petal" mode converter (converts between $\mathrm{TE}_{10}$ rectangular and $\mathrm{TE}_{01}$ circular modes), were not robust enough at higher power levels. Because of this, a major effort is underway to upgrade the existing devices to operate stably at the higher power levels, and also to investigate new components, which have inherently low field enhancement in their design and therefore more robust.

\section{DUAL KLYSTRON TEST}

In order to have sufficient $\mathrm{rf}$ power to stress the critical components beyond that of earlier tests it was necessary to combine the power of two $50 \mathrm{MW}$ klystrons using a 3$\mathrm{dB}$ hybrid (Magic Tee). The drive signals to the two klystrons were phased so that the unused, terminated arm of the hybrid had a minimal signal. The combined power was then fed into the SLED-II 3-dB hybrid as shown in figure 1.

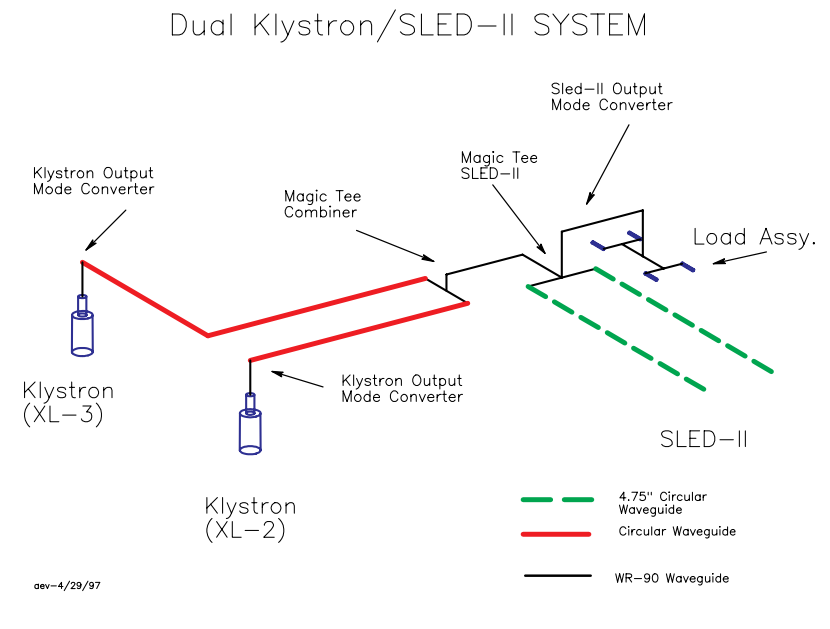

Figure1. Dual Klystron Test Setup

In order to absorb the total power from SLED-II, the output power was split equally into four all-metal choke loads [2] using three Magic Tees operating in parallel. Three water-cooled "grapefruit" loads [3] were used to terminate the unused arm of each Magic Tee. Forward and reflected rf power was measured at the input and output of SLED-II with side-coupled directional couplers and an HP-8990A Peak Power Analyzer. In order to minimize $\mathrm{rf}$ losses (and improve vacuum pumping efficiency), the rf was transported through overmoded circular waveguide in the $\mathrm{TE}_{01}$ low loss mode. Conversion between the circular and rectangular waveguides was accomplished by "Flower Petal" $\mathrm{TE}_{10}-\mathrm{TE}_{01}$ mode converters.

$\mathrm{RF}$ conditioning was initially rather slow because of outgassing in the long lengths of new waveguide, mode

\footnotetext{
*Work supported by Department of Energy contract DE-AC03-76SF00515.
} 
converters and combiner added upstream of the SLED-II Magic Tee. It required approximately 5 weeks of conditioning to reach $180 \mathrm{MW}$. In order to separate the conditioning of the new components from the high power testing of SLED-II, the $180^{\circ}$ phase switching (PSK) of the klystron input signal was initially not used. In this way the output power of SLED-II was kept low (power gain of $\approx 2$ ) while the klystrons were brought up to full power. (a combined power of $\approx 90 \mathrm{MW}$ ). Once this section of the system was conditioned and operated stably, the klystron power was lowered, the PSK was made operatonal and the SLED-II system was operated at its normal 4:1 power gain. At this point essentially all vacuum and rf breakdown activity occurred at the SLED-II output mode converter and Magic Tee. Radiation levels at this mode converter exceeded $10 \mathrm{mR} / \mathrm{hr}$ for output SLED-II power levels of $225 \mathrm{MW}$ and became higher as the power was raised further. After two additional weeks of conditioning, peak power levels of up to $300 \mathrm{MW}$ were measured at pulse repetition rates of 10 pps. (see fig. 2.)

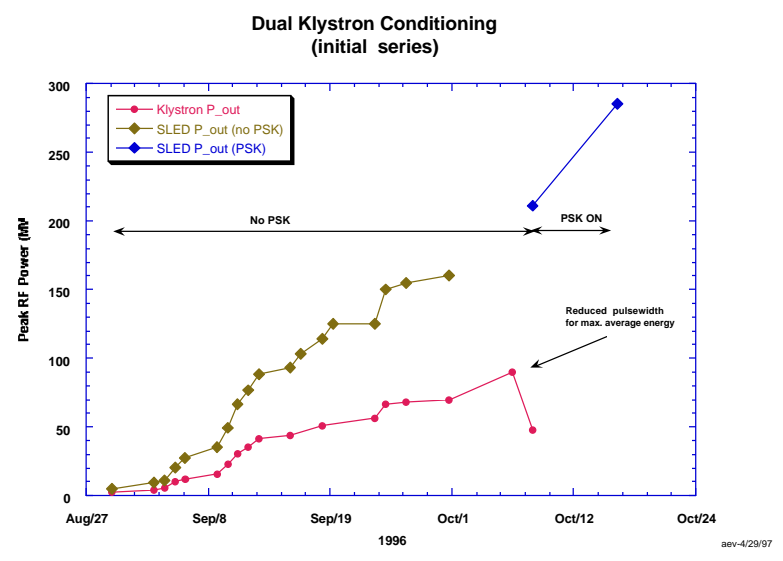

Figure 2. Initial System Conditioning

These power levels, however, could not be maintained for more than a few seconds at a time before rf breakdown occurred. At power levels of $275 \mathrm{MW}$ the system could operate for minutes at a time and below $250 \mathrm{MW}$ the system operated stably. Since most of the breakdown occurred at the output mode converter it was decided to open up the system and investigate the condition of this component. Damage to the device was obvious on initial inspection. The end of the bifurcation in the rectangular guide was severely eroded as was the opposing wall. Since this mode converter was an early model which had no deliberate radius at the bifurcation tip, it was decided to replace the component with a new model which had a $0.8 \mathrm{~mm}$ radiused edge. Testing was then resumed. Progress was rapid until power levels of $220 \mathrm{MW}$ was reached. Above this level, breakdown in the new mode converter limited progress and the peak power level attained was $\approx 280 \mathrm{MW}$. Completely stable operation was only possible at 220-250 MW. After reopening the system, the new mode converter showed damage similar to the first one. In order to test other components in the system it was decided to eliminate the mode converters and connect the load assembly directly to the SLED-II Magic Tee. Repeating the experiment showed that the Magic Tee became the dominant source of breakdown. Surprisingly, the limiting power level was near the other previous values. $\approx 265 \mathrm{MW}$. At this point the experiment was concluded and the entire SLED-II system was inspected. It was found that the SLED-II Magic Tee was badly eroded in two trouble spots; the sharp corners on three sides of the opening into the $\mathrm{E}$ arm and the rounded tip of the single ended matching post in the throat of the four-port junction. No other component showed severe erosion or damage.

\section{HIGH POWER COMPONENTS}

Results of these tests clearly showed a need for improved component performance at the power levels required by NLC. Accordingly, efforts are underway at SLAC to improve the performance of some of the existing key components and to develop new components for NLC which are inherently robust at high peak fields.

\subsection{Modified Components:}

We have developed modifications to the Magic Tee and Flower Petal mode converters, based on the damage observed from tests and from HFSS computer simulations, which have reduced field levels at critical locations. Figure 3 shows a model of the new Magic Tee design.

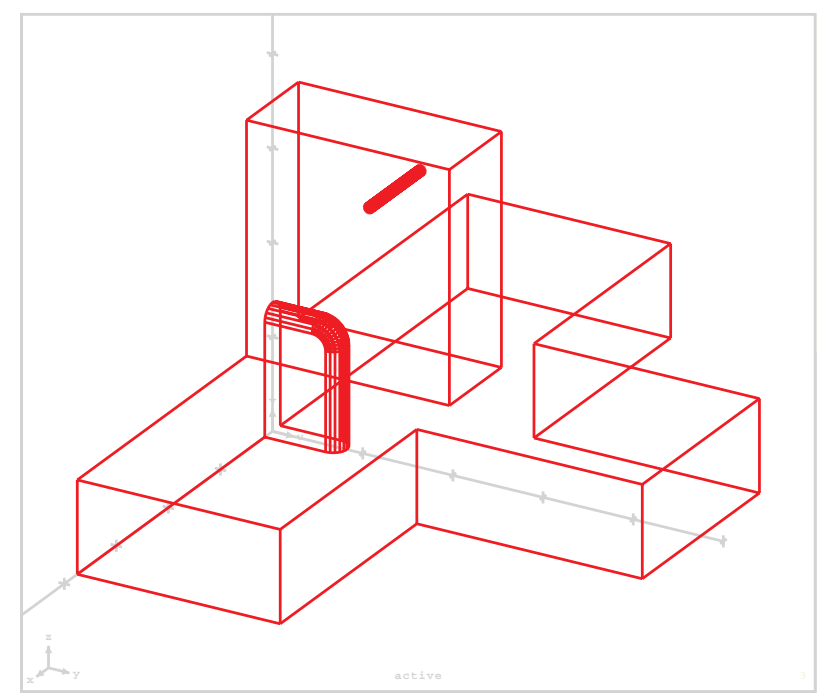

Figure 3. Modified Magic Tee Design

Magic Tee simulations using HFSS [4] indicate that a "fat" fin in the throat and an inductive element in the Earm would result in a reduction in $\mathrm{rf}$ electric field by approximately a factor of two below the original SLAC design. A $1.2 \mathrm{~mm}$ radius was machined on three sides of the waveguide opening into the E-arm reducing the local surface electric field at this other trouble spot. The new Flower Petal mode converter design also has lower fields than the original design, and the region of high field is 
much reduced. The reduction is not as significant as for the Magic Tee, however. The exact breakdown mechanism in the mode converter is not as clear as for the Magic Tee since, even in the original design, the rf electric field enhancement at the erosion locations does not appear particularly high according to HFSS simulatons. It is speculated that poor vacuum and perhaps a multipactor phenomenon involving forward scattered electrons may be taking place but no conclusive analysis has been performed. This new design also improves the vacuum pumping in the critical areas. Cold-testing of the new design has been completed.

\subsection{New Components:}

New, planar devices (H-plane) are also being investigated to provide components with minimum field enhancement. These include a short-slot hybrid (see figure 4) and an array of H-plane three port junctions specially matched to form a planar bridge structure.

The Riblet short slot hybrid has low maximum RF electric field for a given power compared with other fourport hybrid designs. The theory for this device is well documented [5]. High directivity and equal power division have been very difficult to achieve in the past because of critical dimensional tolerances. Precise optimization relied ultimately on cut-and-try machining and the addition of a matching element in the slot region. The success of the design depends on simultaneously controlling the phase advance and match of both the $\mathrm{TE}_{10}$ and $\mathrm{TE}_{20}$ modes in the slot region. The phase advance of these two modes, including the reverse shift due to the slot end effects, must differ by exactly $90^{\circ}$ to give equal power split. At the same time the slot length is very critical to achieve a good match and hence directivity. In

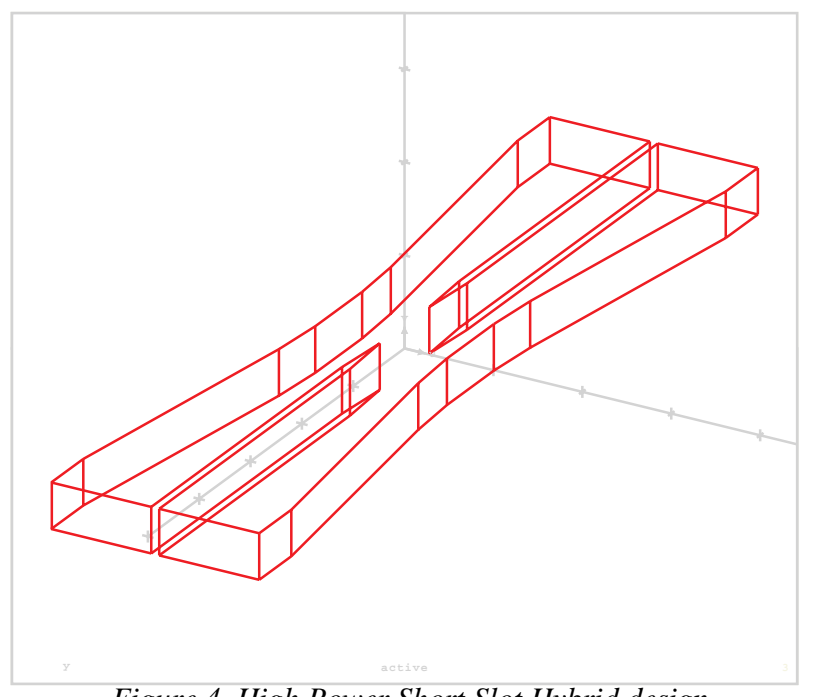

Figure 4. High Power Short Slot Hybrid design

addition, the slot region width must necessarily be narrower than twice the single waveguide width to avoid exciting the TE30 mode.
Today using codes such as HFSS and MAFIA very good directivity, match and power division can be modeled and optimized before cutting any metal. A paper design for a $3 \mathrm{~dB}$ short slot hybrid that requires no matching elements has been completed. See figure 4 .

The maximum RF electric field is somewhat higher than the field in the waveguide ports feeding the device.

A new "wrap-around" mode converter [6] (see figure 5) has also been designed with $40 \%$ lower peak electric fields than the original "Flower Petal". Recent cold-test

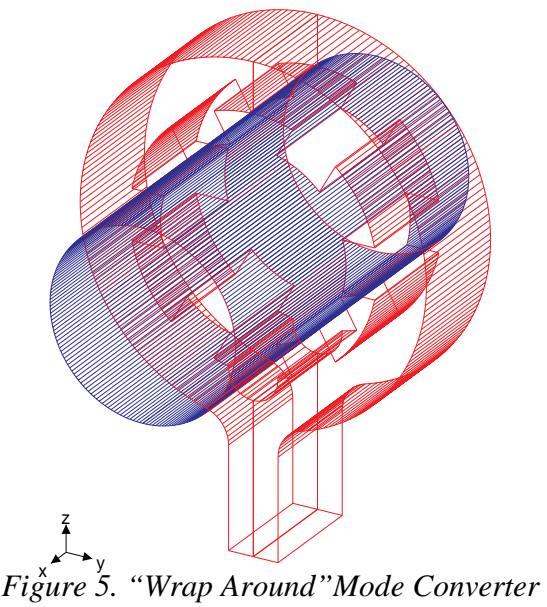

results show excellent performance and also in complete agreement with calculation.

\section{CONCLUSION}

We have designed and are building new high power rf components which, according to simulation, will have lower peak electric field than their existing counterparts in NLCTA and ASTA. These new components are direct replacement parts and should permit higher power operation for these facilities. In addition, we are completing the design of a new family of components which will have intrinsically, the lowest field enhancement possible for a given rectangular waveguide dimension. They will find their application in the NLC.

\section{REFERENCES}

[1] S.G.Tantawi et Al: 'The Next Linear Test Accelerator's RF Pulse Compression and Transmission Systems, 7P.58, PAC'97

[2] Sami G. Tantawi and A. E.Vlieks: 'Compact X-band High Power Load Using Magnetic Stainless Steel', p.2132 Proceedings of the 1995 Particle Accelerator Conference and International Confrence on High Energy Accelerators.

[3] W.R. Fowkes, R.S. Callin, A.E. Vlieks: 'High Power RF Window and Waveguide Component Development and Testing above 100MW at X-Band'. SLAC-PUB-5877, Aug 1992. 3pp., 1992, Proceedings, Linear accelerator Conference, vol. 1 184-186

[4] HFSS is Hewlett-Packard's "High Frequency Structure Simulator" software package.

[5] Riblet,H. J., 'The Short-Slot Hybrid Junction', Proc. I.R.E., 1952, 40, p. 180.

[6] S. Tantawi, K.Ko, V. Srinivas and A.E.Vlieks, 'A Compact High Power $\mathrm{TE}_{01}$ to $\mathrm{TE}_{10}$ Mode Converter., 7P.57, PAC'97 